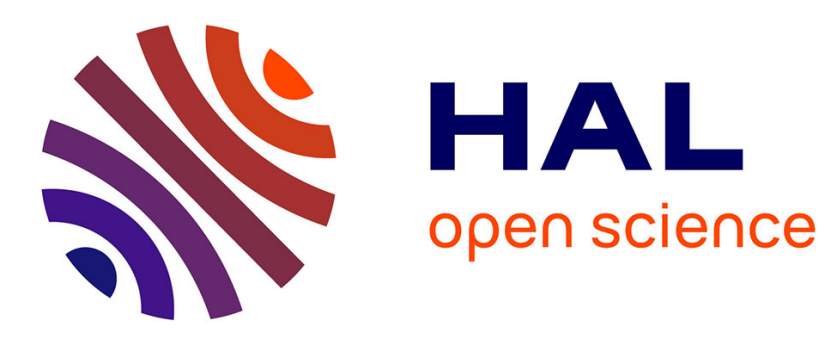

\title{
Mutual Localization and Positioning of Vehicles Sharing GNSS pseudoranges: Sequential Bayesian approach and Experiments
}

\author{
Khaoula Lassoued, Isabelle Fantoni, Philippe Bonnifait
}

\section{To cite this version:}

Khaoula Lassoued, Isabelle Fantoni, Philippe Bonnifait. Mutual Localization and Positioning of Vehicles Sharing GNSS pseudoranges: Sequential Bayesian approach and Experiments. 18th IEEE International Conference on Intelligent Transportation Systems (ITSC 2015), Sep 2015, Las Palmas, Spain. pp.1896-1901, 10.1109/ITSC.2015.307 . hal-01203085

\section{HAL Id: hal-01203085 \\ https://hal.science/hal-01203085}

Submitted on 24 Sep 2015

HAL is a multi-disciplinary open access archive for the deposit and dissemination of scientific research documents, whether they are published or not. The documents may come from teaching and research institutions in France or abroad, or from public or private research centers.
L'archive ouverte pluridisciplinaire HAL, est destinée au dépôt et à la diffusion de documents scientifiques de niveau recherche, publiés ou non, émanant des établissements d'enseignement et de recherche français ou étrangers, des laboratoires publics ou privés. 


\title{
Mutual Localization and Positioning of Vehicles Sharing GNSS pseudoranges: Sequential Bayesian approach and Experiments
}

\author{
Khaoula Lassoued, Isabelle Fantoni and Philippe Bonnifait
}

\begin{abstract}
In many cooperative Intelligent Transportation Systems (ITS) applications, absolute positioning and relative localization are key issues. When vehicles share GNSS positions, there are often non negligible common-mode errors due mainly to GNSS signal propagation and inaccurate ephemeris data. Cooperative observation techniques allow estimating common biases on the measured pseudodistances to correct these errors and to increase absolute positioning and relative localization accuracy. After having studied some structural properties of the problem in its general form, a low computational cooperative tightly-coupled approach is proposed using sequential Kalman filtering and convex data fusion. As a case study, we consider two vehicles, which cooperate and exchange information in such a way that each vehicle can track the partner's position and improves its absolute position by merging common biases estimates. Experimental results are presented to illustrate the performance of the proposed approach in comparison with a classic standalone method.
\end{abstract}

\section{INTRODUCTION}

In many ITS applications, direct wireless communication $\mathrm{V} 2 \mathrm{~V}$ (Vehicle To Vehicle) allows the traffic participants to know where they are with respect to the others even if they can't see the others with their own exteroceptive sensors (e.g. cameras or lidars). But, when using low cost Global Navigation Satellite Systems (GNSS) receivers in complex environments, errors can have a great impact. The effects of these errors can be reduced by considering information exchange. Standalone GNSS positioning is affected by atmospheric and ephemeris errors and also multipath. Our target is to eliminate the common errors and to improve the positioning and localization performance. Positioning means the estimation of the ego position in a fixed working frame and localization refers to the estimation of the relative partners positions in the ego frame. The bias error of standalone GNSS is little observable when loosely coupling the GNSS fixes with Dead Reckoning (DR). In [1], the GPS bias is estimated using the perception of lanes and crosswalks stored in the map data. In [2], the authors used a GPS/DR/map in a tightly coupled mode for autonomous vehicle navigation. Moreover, they proposed an error model to estimate the bias on every pseudorange. $\mathrm{V} 2 \mathrm{~V}$ communication allows taking advantage of the fact that GPS receivers operating in close vicinity and observing the same constellation of satellites have correlated errors. These errors can be largely eliminated when other receivers are taken into consideration as it is done in the differential DGPS technique, when a reference station estimates the errors thanks to its known surveyed position

Sorbonne Universités, Université de Technologie de Compiègne, CNRS, UMR 7253 Heudiasyc, Compiègne, France. and provides this information in the form of corrections to the users located in the vicinity [3]. In [4], a loosely coupled RTK GPS receiver position with DR/laser/magnets sensors is proposed in order to reduce errors in position estimates for an accurate cooperative localization. In the present work, we do not compensate the position estimates but we exploit raw pseudoranges measurements since we consider environments in which the vehicles may not have the same satellites in view. Our approach has some similarities with DGPS but an important difference comes from the fact that the considered mobile GNSS receivers are not precisely located. Two existing main data fusion processes address this issue: centralized and decentralized. The first one is based on a central fusion point or group of points depending on the agents operation in a limited or large environment. Mao et. al [5] proposed a centralized Bayesian algorithm for vehicle positioning and tracking in large environments. However, their algorithm is not able to realize cooperative tracking. The second fusion scheme is based on a decentralized architecture which can also be separated in data exchange or state exchange approaches. In [6], robots exchange relative and absolute positioning information. The cons of this approach are the large quantity of transmitted information. When exchanging global states, this data fusion strategy can significantly reduce the amount of shared data but it has to address the data incest problem (i.e. the result of a repeated use of identical information). Roumeliotis et al. introduced a distributed approach for collective localization based on a centralized EKF [7] and resolved the data incest problem by exchanging only a part of the group state [8] between vehicles. An other approach [9] exploits all the available information in each vehicle and handled private copies of the group knowledge to solve the data incest issue. To do the data fusion, there are different fusion operators depending on the assumptions whether the two estimates to be fused are intercorrelated or not. One can use Simple Convex Combination (SCC), Bar-Shalom/Campo Combination (BCC), Information De-correlation (ID), Linear Minimum Variance Estimate (LMVE) and Covariance Intersection (CI) [10].

In this work, we propose a new formulation of multi-vehicles cooperation using sequential Bayesian estimation and SCC. The novelty of the proposed approach lies in improving ego positioning and mutual localization in between vehicles by sharing GNSS pseudoranges biases. The developed method allows the data fusion of estimated biases in a distributed way with no central fusion node using SCC by assuming that the cross-covariance between two estimates can be ignored. Experimental evaluation using two vehicles is performed to 
face real conditions. Both methods, cooperative and standalone, are compared to illustrate the improvements brought by cooperation.

Section II introduces the system modeling. Section III outlines an observability study of the cooperative localization using biased exteroceptive measurements. The proposed distributed cooperative localization algorithm is described in section IV. Experimental results are presented in section V, with a comparison between the standalone and cooperative methods.

\section{Modeling}

We present here the mathematical models for $n_{r}$ vehicles. Each vehicle $R_{i}$ has to be able of operating autonomously without being reliant on a central processing unit. A fully distributed state estimation method is adopted.

\section{A. Observation model}

The observation model is the distance from each vehicle $i\left(i=\left\{1, \ldots, n_{r}\right\}\right)$ located at coordinates $\boldsymbol{p}_{i}=\left(x_{i}, y_{i}, z_{i}\right)$ to each satellite $j\left(j=1, \ldots, n_{s}\right)$ at position $\boldsymbol{p}_{j} . n_{r}$ and $n_{s}$ denote the number of vehicles and the total number of satellites in view from the vehicles. In a GNSS system, this distance is derived from the time of transmission which is called pseudorange ${ }^{i} \rho^{j}$ [3]. Moreover, the position of every satellite is known with some error $\Delta_{j}=\left(\delta_{x_{j}}, \delta_{y_{j}}, \delta_{z_{j}}\right)$ in the working frame. Eq. (1) shows the expression of ${ }^{i} \rho^{j}$, where $d_{i}=c \delta t_{i}$ is the receiver clock offset ( $\delta t_{i}$ is the receiver clock delay of vehicle $i$ and $c$ is the speed of light):

${ }^{i} \rho^{j}=\sqrt{\left(x_{i}-\left(x_{j}+\delta_{x_{j}}\right)\right)^{2}+\left(y_{i}-\left(y_{j}+\delta_{y_{j}}\right)\right)^{2}+\left(z_{i}-\left(z_{j}+\delta_{z_{j}}\right)\right)^{2}}+d_{i}$

This model can be linearized with $\Delta_{j}$ acting as an additive bias $b^{j}$ on the pseudorange ${ }^{i} \rho^{j}$. Considering:

$$
\left\{\begin{array}{c}
{ }^{i} \rho_{\Delta}^{j}=\sqrt{\left(x_{i}-\left(x_{j}+\delta_{x_{j}}\right)\right)^{2}+\left(y_{i}-\left(y_{j}+\delta_{y_{j}}\right)\right)^{2}+\left(z_{i}-\left(z_{j}+\delta_{z_{j}}\right)\right)^{2}} \\
{ }_{i} \rho_{\text {real }}^{j}=\sqrt{\left(x_{i}-x_{j}\right)^{2}+\left(y_{i}-y_{j}\right)^{2}+\left(z_{i}-z_{j}\right)^{2}}+d_{i}
\end{array}\right.
$$

Eq. (1) becomes:

$$
{ }^{i} \rho^{j}={ }^{i} \rho_{\Delta}^{j}+d_{i}
$$

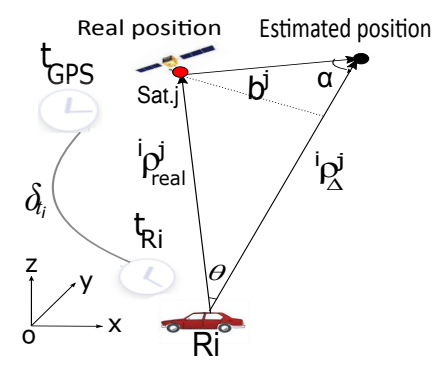

Fig. 1: GNSS pseudoranges

From Fig. 1 we have :

$$
\cos \alpha=\frac{{ }^{i} \rho_{\Delta}^{j}-{ }^{i} \rho_{\text {real }}^{j} \cos \theta}{b^{j}}
$$

The pseudoranges ${ }^{i} \rho_{\Delta}^{j}$ and ${ }^{i} \rho_{\text {real }}^{j}$ from satellite $j$ to vehicle $i$ are different from the distance $b^{j}$ between the real position of satellite and the erroneous one. The two lines of sight ${ }^{i} \rho_{\Delta}^{j}$ and ${ }^{i} \rho_{\text {real }}^{j}$ are nearly parallel to each other. It means that $\theta \approx 0$ and then $\alpha \approx 0$. Taking Eq. (3) while considering $\alpha \approx 0$ and $\theta \approx 0$ we get:

$$
{ }^{i} \rho_{\Delta}^{j}={ }^{i} \rho_{\text {real }}^{j}+b^{j}
$$

Using Eq. (4), Eq. (1) becomes:

$$
{ }^{i} \rho^{j}=\sqrt{\left(x_{i}-x_{j}\right)^{2}+\left(y_{i}-y_{j}\right)^{2}+\left(z_{i}-z_{j}\right)^{2}}+b^{j}+d_{i}
$$

where $b^{j}$ is the bias of satellite $j$. In section $\mathrm{V}$, we will add a term ${ }^{i} \beta^{j}$, in order to take into account the measurement noise. The observation model presented in Eq. (5) being similar for each vehicle, the pseudoranges vector is written as:

$$
{ }^{i} Y=\left[\begin{array}{llll}
{ }^{i} \rho^{1} & { }^{i} \rho^{2} & \ldots & { }^{i} \rho^{n_{s}}
\end{array}\right]^{T}
$$

\section{B. Evolution model}

A classical unicycle model $f$ is used to predict the DR position and orientation of the vehicles. The receiver clock offset $d_{i}$ and its drift $\dot{d}_{i}$ have also to be taken into account. Each vehicle is equipped with a yaw rate gyro and wheel encoders to measure the speed and the angular velocity, which constitute an input vector ${ }^{i} U_{k}=\left[\begin{array}{cc}v_{i, k} & \omega_{i, k}\end{array}\right]^{T}$. The evolution model of vehicle $i$ and an auto-regressive (AR) biases model at each sample $k$ are described by:

$$
f=\left\{\begin{aligned}
x_{i, k} & =x_{i, k-1}+T_{e} v_{i, k-1} \cos \psi_{i, k-1} \\
y_{i, k} & =y_{i, k-1}+T_{e} v_{i, k-1} \sin \psi_{i, k-1} \\
z_{i, k} & =z_{i, k-1} \\
\psi_{i, k} & =\psi_{i, k-1}+T_{e} \omega_{i, k-1} \\
d_{i, k} & =d_{i, k-1}+T_{e} \dot{d}_{i, k-1}+\alpha_{k}^{d} \\
\dot{d}_{i, k} & =\dot{d}_{i, k-1}+\alpha_{k}^{d} \\
b_{k}^{j} & =a b_{k-1}^{j}+\alpha_{k}^{b_{j}}
\end{aligned}\right.
$$

where $T_{e}$ is the sampling period, $\psi_{i}$ is the orientation angle of the vehicle, $a \in[0,1]$ is the inverse of the correlation time, $\alpha_{k}^{d}, \alpha_{k}^{\dot{d}}$ and $\alpha_{k}^{b_{j}}$ are respectively the noises corresponding to the receiver clock offset $d_{i}$, its drift $\dot{d}_{i}$ and to the bias $b^{j}$ at each sampling time $k$. In the following, $N$ denotes the covariance matrix of the measurements noise $\gamma_{k}=$ $\left[\begin{array}{ll}\gamma_{k}^{v} & \gamma_{k}^{\omega}\end{array}\right]^{T}$ of $U_{k}$, and $Q$ denotes the covariance matrix of the model noise $\alpha_{k}=\left[\begin{array}{lllll}\alpha_{k}^{b_{1}} & \ldots & \alpha_{k}^{b_{2}} & \alpha_{k}^{d} & \alpha_{k}^{d}\end{array}\right]^{T}$.

\section{OBSERVABILITY CONSIDERATIONS}

Let consider simple and double difference observable combinations to remove biasesand clock offsets from the state. This allows to study the observability of the positions of the cooperative system made of $n_{r}$ vehicles. Let $i=1, \ldots, n_{r}$ be the index of the vehicles, $j=1, \ldots, n_{s}$ the index of common satellites in between the vehicles and ${ }^{i} R^{j}$ the geometric distance between vehicles and satellites. The single difference between two receivers requires at least one common visible satellite and 2 receivers. Taking the single difference, the common bias $b^{j}$ can be effectively removed:

$$
S D_{(1,2)}^{j}={ }^{1} \rho^{j}-{ }^{2} \rho^{j}=\left({ }^{1} R^{j}-{ }^{2} R^{j}\right)+\left(d_{1}-d_{2}\right)
$$


Considering another $k$ satellite, the double difference is the difference of single differences considering at least 2 satellites $\{k, j\}$ and two vehicles. With a double difference, the errors due to the receivers clocks $\left(d_{k}\right.$ and $\left.d_{j}\right)$ can be canceled:

$$
\begin{aligned}
D D_{(1,2)}^{j k} & =S D_{(1,2)}^{j}-S D_{(1,2)}^{k}={ }^{1} \rho^{j}-{ }^{2} \rho^{j}-{ }^{2} \rho^{k}+{ }^{2} \rho^{k} \\
& ={ }^{1} R^{j}-{ }^{2} R^{j}-{ }^{2} R^{k}+{ }^{2} R^{k}
\end{aligned}
$$

Now, the state observability of a cooperative system is studied using the $D D$ measurements (Eq. 9). The system is observable if one can find its unknown variables. We have to find the minimal number $n_{s}$ of satellites such that the system is observable. To obtain at least one $D D$ measurement, we need $n_{r} \geq 2$ and $n_{s} \geq 2$.

$\boldsymbol{p}_{i}$ being the position of each vehicle $i$, the state vector of the cooperative system is:

$$
{ }^{i} \boldsymbol{x}=\left[\begin{array}{llll}
\boldsymbol{p}_{1} & \boldsymbol{p}_{2} & \cdots & \boldsymbol{p}_{n_{r}}
\end{array}\right]^{T}
$$

The number $n_{x}$ of unknown variables of the system ${ }^{i} \boldsymbol{x}$ are the coordinates of each robot $i$ :

$$
n_{x}=3 . n_{r}
$$

Let $n_{m}$ be the number of available $D D$ measures (Eq. 9). Clearly to solve this problem one must have:

$$
n_{m} \geq n_{x}
$$

$n_{m}, n_{r}$ and $n_{s}$ are linked by the following expression where $C_{2}^{n_{s}}$ and $C_{2}^{n_{r}}$ are the binomial coefficients (e.g. combination of 2 satellites from $n_{s}$ satellites respectively combination of 2 robots from $n_{r}$ ) :

$$
n_{m}=C_{2}^{n_{s}} \cdot C_{2}^{n_{r}}
$$

For instance, with 2 vehicles one has $1 D D$ with 2 satellites and $6 D D$ with 4 satellites and $105 D D$ with 15 satellites and with 4 vehicles one has $18 D D$ with 3 satellites.

Using Eq. (11) and (13), the inequality in (12) becomes:

$$
C_{2}^{n_{s}} \cdot C_{2}^{n_{r}} \geq 3 . n_{r}
$$

By taking the expressions $C_{2}^{n_{s}}=\frac{n_{s}\left(n_{s}-1\right)}{2}$ and $C_{2}^{n_{r}}=$ $\frac{n_{r}\left(n_{r}-1\right)}{2}$, we can further simplify Eq. (14) as a second-order polynomial in $n_{s}$ as follows:

$$
f\left(n_{s}\right)=n_{s}^{2}-n_{s}-a \geq 0
$$

where $a=\frac{12}{n_{r}-1} \geq 0,\left(n_{r}-1 \geq 1\right.$ as $\left.n_{r} \geq 2\right)$. Eq. (15) has two solutions and one of them is rejected since it is negative. The minimal required number of common satellites $n_{s}$ between vehicles such that the system is observable is $\frac{1+\sqrt{1+4 a}}{2}$.

As a conclusion of the relation between the solution $n_{s}$ and the number of vehicles $n_{r}$, in order to observe the positions of the vehicles of the system (10) one must have: If $n_{r}=$ $2 \Rightarrow n_{s} \geq 4$, if $3 \leq n_{r} \leq 6 \Rightarrow n_{s} \geq 3$ and if $n_{r} \geq 7 \Rightarrow$ $0<a \leq 2 \Rightarrow 1<S_{1} \leq 2 \Rightarrow n_{s} \geq S_{1}>1 \Rightarrow n_{s} \geq 2$. This conclusion shows that as we increase the number of vehicles, we increase the number of measurements. Then, we can reduce the minimal required number of satellites $n_{s}$.
At this stage, we have the minimal condition to estimate the position of every vehicle.

Now, if we consider the complete state of the cooperative system composed of positions, biases and receiver clock offsets, we can show that the biases and the clock offsets are also observable. Since we know the position of every vehicle and the pseudo-distance measurement, the only unknown variables in Eq. (5) are the bias and the clock offset. In the worse case with 2 vehicles and 4 satellites in view, we get a non singular linear system of 8 equations ( 8 pseudoranges measurements and 8 estimated positions) and 6 unknowns (4 biases and 2 clocks terms) and therefore the biases and the clock offsets are observable.

\section{BAYESIAN COOPERATIVE ESTIMATION}

Suppose the state to be estimated is a finite dimensional random vector $x$ with mean $\bar{x}$ and covariance $P$. Suppose that $n$ estimates $\hat{x}_{i}$ have to be merged with their error covariance given by $P_{i}$. The distributed fusion problem is to estimate an "optimal" estimate $\hat{x}$ from the available estimates. The SCC fusion algorithm is given by Eq. (16), where the error covariance and the state estimate are

$$
\left\{\begin{array}{c}
P^{-1}=\sum_{i=1}^{n} P_{i}^{-1} \\
\hat{x}=P \cdot\left(\sum_{i=1}^{n} P_{i}^{-1} \cdot \hat{x}_{i}\right)
\end{array}\right.
$$

In each agent $R_{i}$, we have an ego part identified by the index ego and a part dedicated to the tracking of the others identified by the index $o$. Let $R_{i}$ be the ego vehicle in the group of $n_{r}$ vehicles. The index of the other vehicles $R_{o_{j}}$ is $j=\{1, \ldots, M\}$ with $j \neq i$ and $M=n_{r}-1$ is the number of other vehicles in the group. This section describes the algorithm running in $R_{i}$. The same algorithm is executed in the other vehicles $R_{o_{j}}$.

${ }^{i} \boldsymbol{x}_{\text {ego }}=\left[\begin{array}{lll}{ }^{i} \boldsymbol{q} & { }^{i} \boldsymbol{b} & { }^{i} \boldsymbol{\xi}\end{array}\right]^{T}$ is the ego state of $R_{i}$ estimated in $R_{i}$, where ${ }^{i} \boldsymbol{q}=\left[\begin{array}{llll}x_{i} & y_{i} & z_{i} & \psi_{i}\end{array}\right]^{T}$ is the pose of $R_{i}$ estimated in $R_{i}$ in the absolute working frame, ${ }^{i} \boldsymbol{b}=$ $\left[b^{1}, \ldots, b^{n_{s}}\right]^{T}$ are the biases of all $n_{s}$ satellites in view estimated in $R_{i}$ and ${ }^{i} \boldsymbol{\xi}=\left[\begin{array}{ll}d_{i} & \dot{d}_{i}\end{array}\right]^{T}$ are the inner variables of $R_{i}$ containing respectively the clock offset $d_{i}$ and its drift $\dot{d}_{i}$. ${ }^{i} P_{\text {ego }}=\left[\begin{array}{ccc}P_{\boldsymbol{q}} & P_{\boldsymbol{q}, \boldsymbol{b}} & P_{\boldsymbol{q}, \boldsymbol{\xi}} \\ P_{\boldsymbol{q}, \boldsymbol{b}} & P_{\boldsymbol{b}} & P_{\boldsymbol{b}, \boldsymbol{\xi}} \\ P_{\boldsymbol{q}, \boldsymbol{\xi}} & P_{\boldsymbol{b}, \boldsymbol{\xi}} & P_{\boldsymbol{\xi}}\end{array}\right]$ is the ego error covariance, ${ }^{i} \boldsymbol{u}_{e g o}=\left[\begin{array}{ll}v_{i} & w_{i}\end{array}\right]^{T}$ is the ego input of $R_{i}$, where $v_{i}$ and $w_{i}$ are respectively the linear speed and the angular rate measurements. ${ }^{i} \boldsymbol{q}_{o}=\left[{ }^{i} \boldsymbol{q}_{o_{1}}^{T}, \ldots,{ }^{i} \boldsymbol{q}_{o_{M}}^{T}\right]^{T}$ is the pose of other vehicles estimated in $R_{i},{ }^{i} \boldsymbol{u}_{o}=\left[{ }^{i} \boldsymbol{u}_{o_{1}}^{T}, \ldots,{ }^{i} \boldsymbol{u}_{o_{M}}^{T}\right]^{T}$ is the input of $R_{o}$ composed of ${ }^{i} \boldsymbol{u}_{o_{j}}=\left[\begin{array}{ll}v_{o_{j}} & w_{o_{j}}\end{array}\right]^{T}$ which contains the linear speed and the angular rate measures of each $R_{o_{j}} .{ }^{i} P_{\boldsymbol{q}, o}=\left[{ }^{i} P_{\boldsymbol{q}, o_{1}}, \ldots,{ }^{i} P_{\boldsymbol{q}, o_{M}}\right]^{T}$ and ${ }^{i} P_{\boldsymbol{b}, o}=$ $\left[{ }^{i} P_{\boldsymbol{b}, o_{1}}, \ldots,{ }^{i} P_{\boldsymbol{b}, o_{M}}\right]^{T}$ are the parts of the errors covariance matrix depending respectively on $q$ and $b^{j}$ of $R_{o_{j}}$.

Fig. 2 specifies the algorithm of the data fusion for agent $R_{i}$. It involves 4 stages: prediction/track, update, communication and fusion. 
Stage 1: The agent $R_{i}$ predicts its ego state using the evolution model in Eq. (6) and Eq. (7) and its DR measurements. Acquisition is done at high rate (typically, $100 \mathrm{~Hz}$ ). Moreover, it tracks the other vehicles using the last known input of the others. The state evolution of the group can be modeled by the function $f_{\text {track }}$ according to Eq. (17):

$$
\begin{aligned}
{ }^{i} \widehat{\boldsymbol{q}}_{o_{j}}= & f_{\text {track }}\left({ }^{i} \widehat{\boldsymbol{q}}_{o_{j}},{ }^{i} \boldsymbol{u}_{o_{j}}\right) \\
= & \left\{\begin{array}{c}
x_{o_{j}, k}=x_{o_{j}, k-1}+T_{e} v_{o_{j}, k-1} \cos \psi_{o_{j}, k-1} \\
y_{o_{j}, k}=y_{o_{j}, k-1}+T_{e} v_{o_{j}, k-1} \sin \psi_{o_{j}, k-1} \\
z_{o_{j}, k}=z_{o_{j}, k-1} \\
\psi_{o_{j}, k}=\psi_{o_{j}, k-1}+T_{e} \omega_{o_{j}, k-1}
\end{array}\right.
\end{aligned}
$$

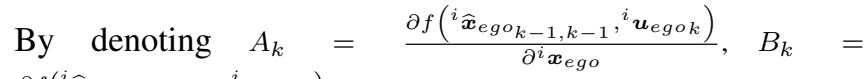
$\frac{\partial f\left({ }^{i} \widehat{\boldsymbol{x}}_{e g o k-1, k-1},{ }^{i} \boldsymbol{u}_{e g o k}\right)}{\partial^{i} \boldsymbol{u}_{e g o}}$, the EKF solver in stage 1 is described by Algorithm 1 and the track process by Algorithm 2.

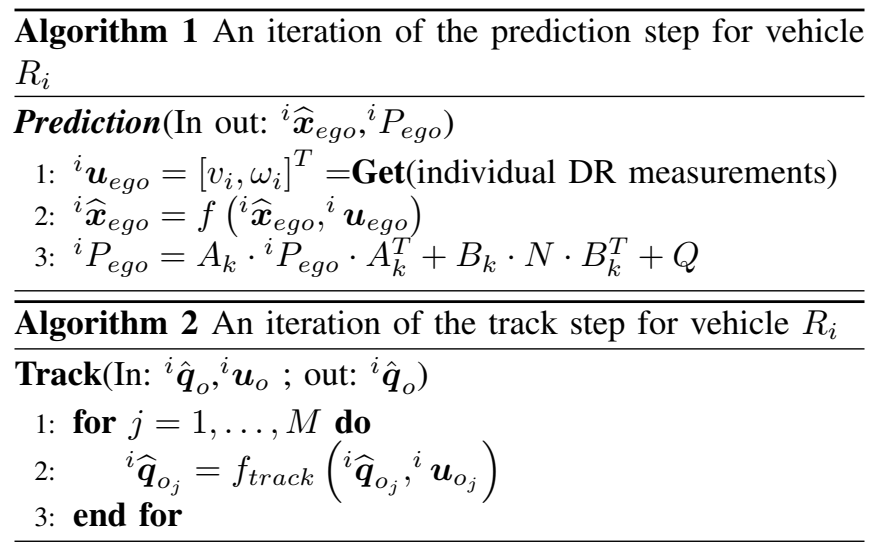

$\underline{\text { Stage } 2}$ : It consists in updating the state ${ }^{i} \boldsymbol{x}_{\text {ego }}$ with respect to GNSS measurements. The acquisition is done at lower rate (for example $5 \mathrm{~Hz}$ when using a classical GPS receiver). In order to reject outliers when the GNSS measurements are made available, a gating process on the measurements of every satellite is adopted. Let $j$ be the index of exteroceptive measurements with $j=\left\{1, \ldots, n_{m}\right\}$. For every pseudorange measurement: Check that the $S N R$ (Signal to Noise Ratio) of satellite is high enough (e.g. $35 \mathrm{dBHz}$ ) and that the Mahalanobis distance $D_{j}$ depending on the innovation is smaller than a threshold. The EKF solver in stage 2 is defined by Algorithm 3. The process is time-triggered with the DR sensors which have the highest rate $(100 \mathrm{~Hz})$. The latency of the GNSS measurements can be neglected.

Stage 3: When a communication is available the cooperation can be done by sharing the following information at each time-stamp $k$ as it is detailed in Algorithm 4:

- $R_{i}$ broadcasts dataS to the other vehicles $:^{i} \boldsymbol{u}_{\text {ego }}$ ( the DR input at time $k$ of $\left.R_{i}\right),{ }^{i} \boldsymbol{q}_{\text {ego }}$ and ${ }^{i} P_{\boldsymbol{q}, \text { ego }}$ (sub-vector and sub-matrix corresponding to $\boldsymbol{q}$ in ${ }^{i} \boldsymbol{x}_{\text {ego }}$ and $\left.{ }^{i} P_{\text {ego }}\right),{ }^{i} \boldsymbol{b}_{\text {ego }}$ and ${ }^{i} P_{\boldsymbol{b} \text {,ego }}$ (sub-vector and sub-matrix corresponding to $b^{j}$ in ${ }^{i} \boldsymbol{x}_{e g o}$ and ${ }^{i} P_{e g o}$ ) and ${ }^{i} i d$ (the identifier of ego vehicle).

- $R_{i}$ receives from each other vehicles $R_{o_{j}}$ the following dataR: ${ }^{o_{j}} \boldsymbol{u}_{\text {ego }}$ (the DR input at time $k$ of $R_{o_{j}}$ ), ${ }^{o_{j}} \boldsymbol{q}_{\text {ego }}$ and ${ }^{o_{j}} P_{\boldsymbol{q}, \text { ego }}$ (sub-vector and sub-matrix corresponding to $\boldsymbol{q}$ in ${ }^{o_{j}} \boldsymbol{x}_{\text {ego }}$ and ${ }^{o_{j}} P_{\text {ego }}$ ), ${ }^{o_{j}} \boldsymbol{b}_{\text {ego }}$ and ${ }^{o_{j}} P_{\boldsymbol{b}, \text { ego }}$ (subvector and sub-matrix corresponding to $b^{j}$ in ${ }^{o_{j}} \boldsymbol{x}_{e g o}$ and $\left.{ }^{o_{j}} P_{\text {ego }}\right)$ and ${ }^{o_{j}} i d$ (ID of other vehicles).
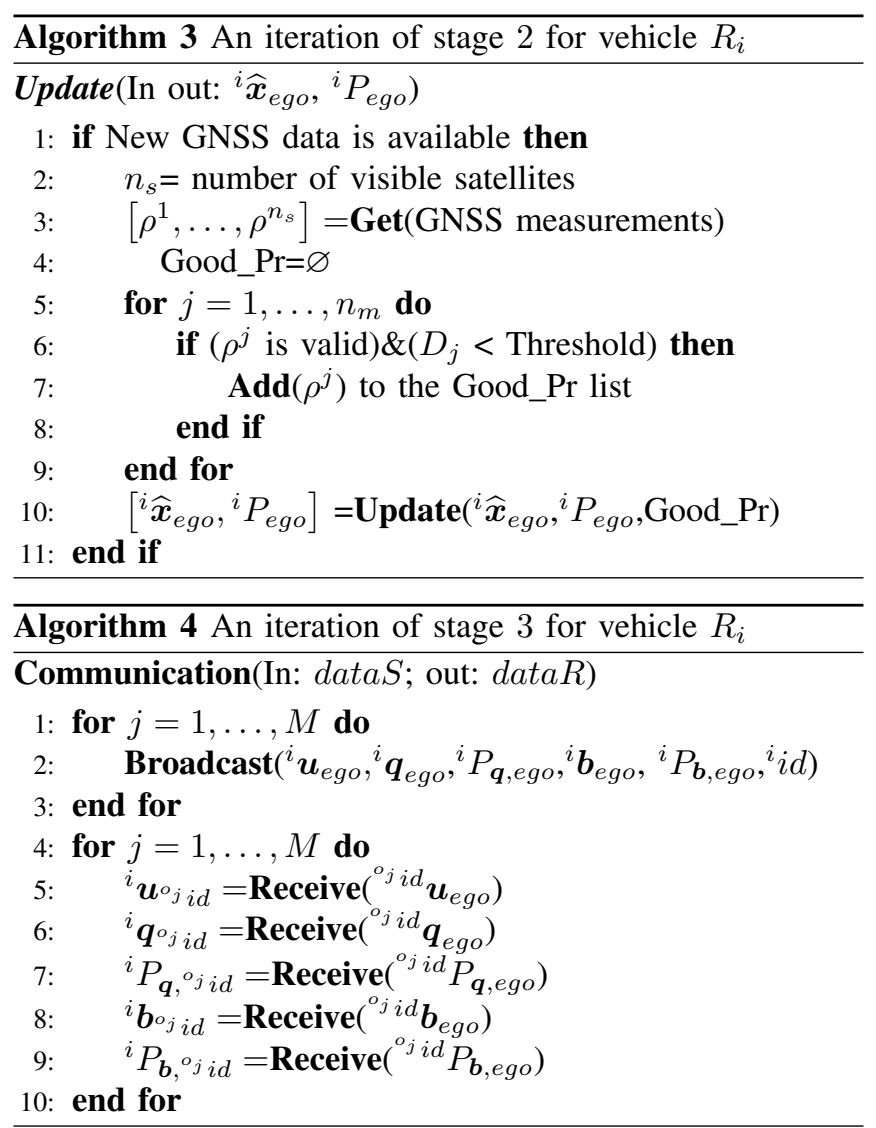

Stage 4: The track update consists in replacing ${ }^{i} \boldsymbol{q}_{o},{ }^{i} P_{\boldsymbol{q}, o}$ by ${ }^{o} \boldsymbol{q}_{\text {ego }},{ }^{o} P_{\boldsymbol{q}, \text { ego }}$ at time-stamp $k$ (Algorithm 5). The bias update corresponds to the fusion of the received estimated biases of the other vehicles ${ }^{o} \boldsymbol{b}_{\text {ego }}$ and the $R_{i}$ ego estimated biases ${ }^{i} \boldsymbol{b}_{\text {ego }}$ using the SCC fusion algorithm. For example, consider the fusion process in vehicle $R_{i}$. By denoting by ${ }^{i} \boldsymbol{b}_{c}$ the result of the fusion of the biases, by ${ }^{i} P_{b_{c}}$ the result of the errors covariance of biases fusion and by $n_{M}$ the number of detected $R_{o_{j}}$, we get Algorithm 6. We can now write the main cooperative Algorithm 7.

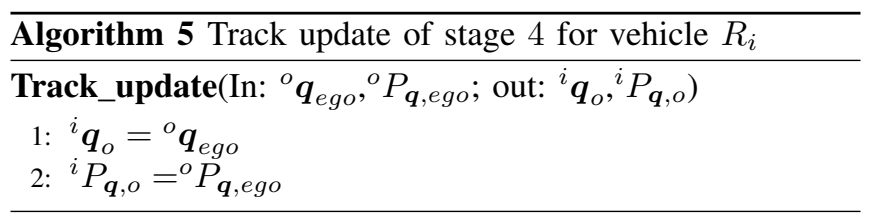

\section{EXPERIMENTAL RESULTS}

The results of two scenarios, i.e., standalone and cooperative, are reported here to quantify the performance gain due to the cooperation. In this work, we tested our approach only with two vehicles. Two automotive experimental vehicles performed different trajectories near the Heudiasyc laboratory. A low-cost U-blox $4 T$ GPS receiver providing pseudoranges measurements at $5 \mathrm{~Hz}$ was used in every vehicle. Each vehicle was equipped with a Polarx Septentrio in RTK mode 


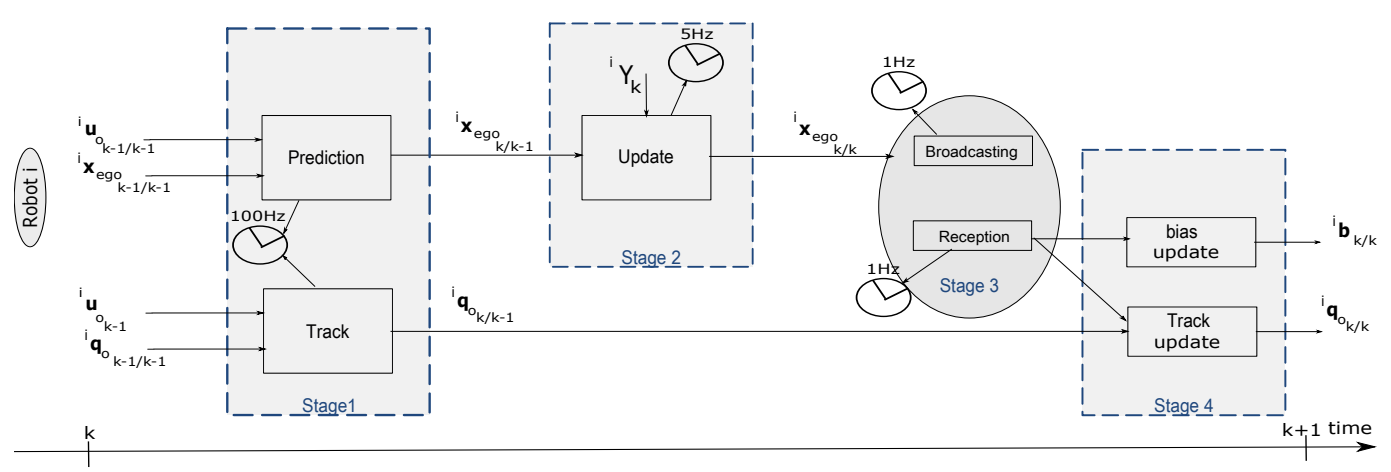

Fig. 2: Main stages of the estimation process
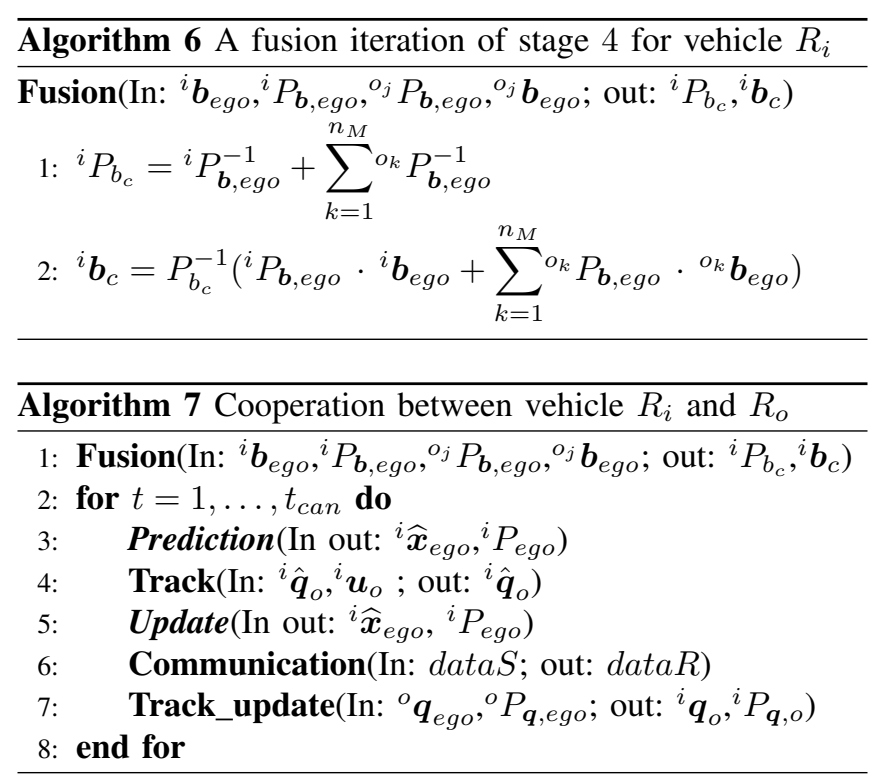

to obtain the ground truth data and a CAN-bus gateway to get the measured input $\boldsymbol{u}_{\text {ego }}=\left[\begin{array}{ll}v & w\end{array}\right]^{T}$ at $100 \mathrm{~Hz}$ rate. 10 satellites were in view during the acquisition and 6 of them were at least in common, which satisfies the condition of the observability study in III. The GPS satellite visibility was sometimes very constrained due to the buildings near the test area. Positioning and localization performance is studied in terms of the 2D pose errors. Fig. 3 and 4 show the absolute Horizontal Positioning Errors (HPE) of $R_{1}$ and $R_{2}$ for the cooperative $(\mathrm{C})$ and the standalone (S) methods. We can notice that the $\mathrm{C}$ method has reduced the confidence domain size and the HPE, especially for the vehicle $R_{1}$ despite the integrity lack in some samples compared to the $\mathrm{S}$ method. Fig. 5 shows the relative localization errors for both methods. A gain of accuracy of the order of several meters is observed.

Fig. 6 shows the cumulative distribution of HPE of $R_{1}$ for the $\mathrm{C}$ and $\mathrm{S}$ methods. It is noticeable that the cooperative method improves the accuracy compared to standalone since the 95th percentiles of the $\mathrm{C}$ and $\mathrm{S}$ methods are respectively $8.56 \mathrm{~m}$ and $9.87 \mathrm{~m}$. Fig. 7 shows the Cumulative Distribution Function of the confidence domain size of the HPE of $R_{1}$ by the $\mathrm{S}$ and $\mathrm{C}$ methods. The $95 t h$ percentiles of the cooperative and standalone methods are respectively $5.67 \mathrm{~m}^{2}$
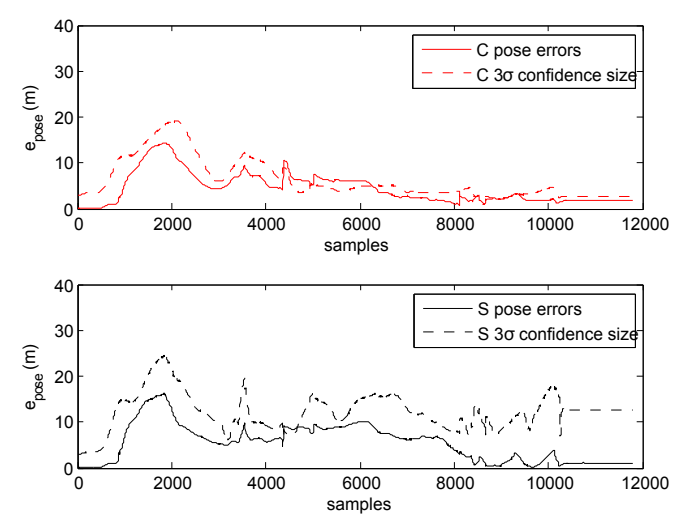

Fig. 3: Absolute Horizontal Positioning Errors of $R_{1}$.
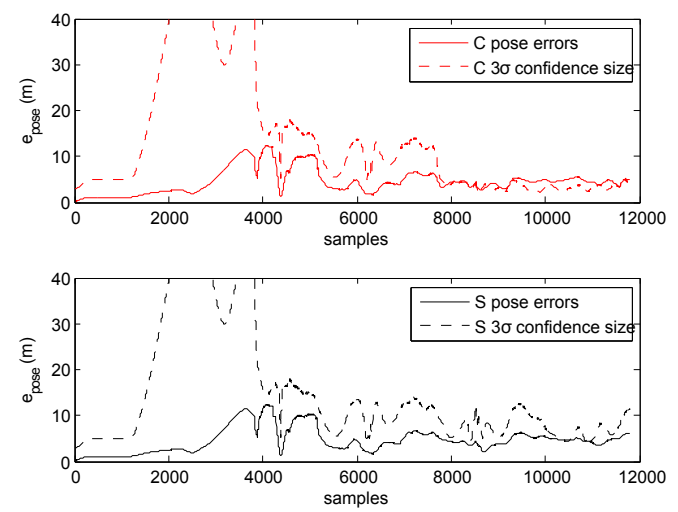

Fig. 4: Absolute Horizontal Positioning Errors of $R_{2}$.

and $6.61 \mathrm{~m}^{2}$. The cooperative approach also improves the $\mathrm{S}$ method in terms of confidence. A net improvement is obtained for the vehicle $R_{1}$ where HPE standard deviation is reduced from $4.25 \mathrm{~m}$ to $3.65 \mathrm{~m}$ using our approach compared to the $\mathrm{S}$ method. Concerning the vehicle $R_{2}$ the improvement of HPE is not as noticeable as in $R_{1}$, but the confidence domain size is reduced.

The accuracy improvement of the relative localization is obtained thanks to the fusion of biases estimates. Especially, the error average and error standard deviation are reduced respectively from $7.85 m$ and $4.2 m$ to $6.5 m$ and $3.9 m$ using the cooperative approach.

The bias on every pseudorange is initialized at zero. For each subplot of Fig. 8 the abscissa expresses time in seconds, the ordinate gives every estimated bias $b^{j}$ on meters where 

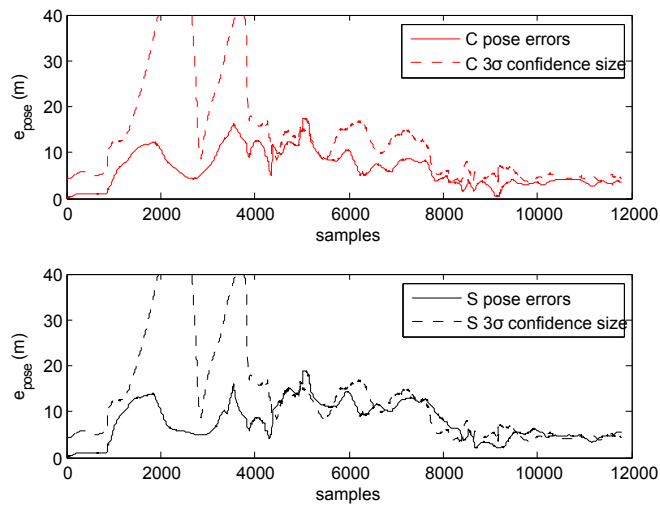

Fig. 5: Relative Horizontal localization Errors

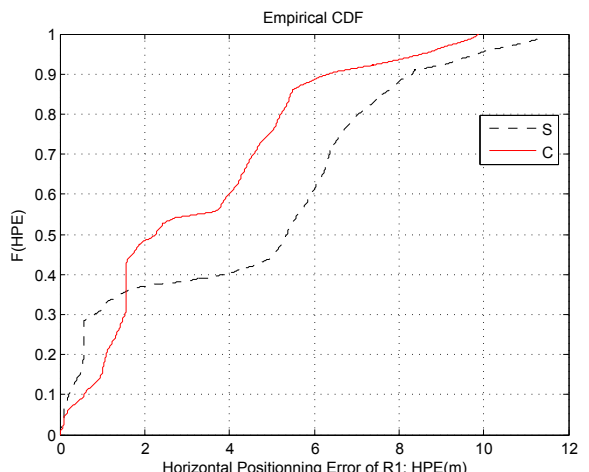

Fig. 6: Cumulative Distribution of the HPE of $R_{1}$

$j=1, \ldots, n_{s}$. The measurements of satellites 17 and 27 became available after $76 \mathrm{~s}$ and after $116 \mathrm{~s}$ for satellite 28 .

\section{CONCLUSION}

This paper has described a cooperative sequential Bayesian approach for positioning and localization of cooperative vehicles. An observability analysis has been developed to show that is possible to estimate the biases of the measurements even if no vehicle is accurately located. Cooperation clearly improves absolute positioning and mutual localization. The use of an easy-to-implement SCC fusion operator proves that the method gives better results in terms of accuracy compared to the classical standalone method. The effect of the use of SCC on the confidence estimation has been also studied. Indeed, this data fusion operator is not robust to the data incest problem since it reuses the fused biases estimates in a sequential way. We have noticed that the cooperative method looses sometimes its consistency as shown by the experiments. Therefore, ongoing work is on the implementation of set-membership methods to deal with this issue [11] to get reliable confidence domains.

\section{ACKNOWLEDGMENT}

This work was carried out within the framework of the Equipex ROBOTEX (Reference ANR-10- EQPX-44-01), which was funded by the European Regional Development Fund.

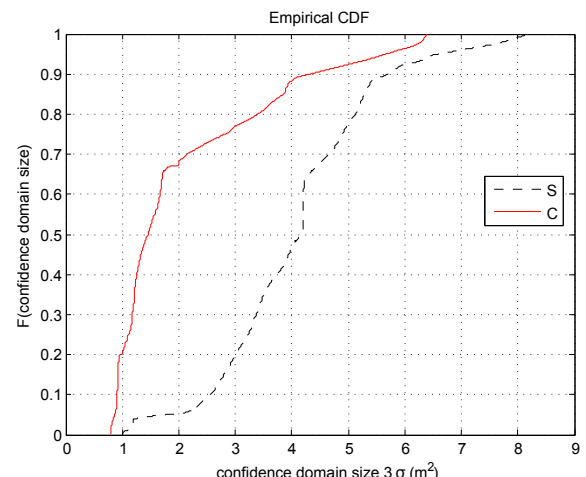

Fig. 7: Cumulative Distribution of the confidence domain of $R_{1}$
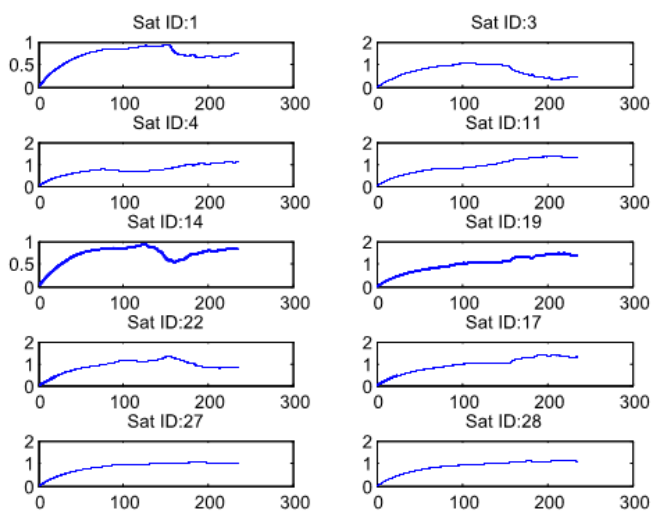

Fig. 8: Estimated bias on every pseudorange

\section{REFERENCES}

[1] K. Jo, K. Chu, and M. Sunwoo. GPS-bias correction for precise localization of autonomous vehicles. In IEEE Intelligent Vehicles Symposium (IV), pages 636-641, Australia, 2013.

[2] Z. Tao and Ph. Bonnifait. Tightly coupling GPS with lane markings for autonomous vehicle navigation. In 17th IEEE International Conference on Intelligent Transportation Systems (ITSC), pages 439_ 444, Qingdao, China, Oct 2014.

[3] Kaplan E. D. and C. Hegarty. Understanding GPS: Principles and Applications. Second Edition, Artech House, 2005.

[4] R. Parafita L.C. Bento and U. Nunes. Inter-vehicle sensor fusion for accurate vehicle localization supported by $\mathrm{v} 2 \mathrm{v}$ and $\mathrm{v} 2 \mathrm{i}$ communications. In 15th International IEEE Conference on Intelligent Transportation Systems (ITSC), pages 907-914, USA, 2012.

[5] S. Mao, S. Worrall, and E. Nebot. Probabilistic long-term vehicle motion prediction and tracking in large environments. IEEE Transactions on Intelligent Transportation Systems, 14(2):539-552, June 2013.

[6] R. Madhavan, K. Fregene, and L.E. Parker. Distributed heterogeneous outdoor multi-robot localization. In IEEE International Conference on Robotics and Automation (ICRA), volume 1, pages 374-381, 2002.

[7] S.I. Roumeliotis and G.A. Bekey. Distributed multirobot localization. IEEE Transactions on Robotics and Automation, 18(5):781-795, 2002.

[8] S.I. Roumeliotis and I.M. Rekleitis. Analysis of multirobot localization uncertainty propagation. In IEEE/RSJ International Conference on Intelligent Robots and Systems, volume 2, pages 1763-1770, 2003.

[9] R. Aufrere, N. Karam, F. Chausse, and R. Chapuis. A state exchange approach in real conditions for multi-robot cooperative localization. In IEEE/RSJ International Conference on Intelligent Robots and Systems (IROS), pages 4346-4351, Taipei, Taiwan, 2010.

[10] CY. Chong and S. Mori. Convex combination and covariance intersection algorithms in distributed fusion. In 4th International Conference on Information Fusion, Canada, 2001.

[11] K. Lassoued, O. Stanoi, Ph. Bonnifait, and I. Fantoni. Mobile robots cooperation with biased exteroceptive measurements. In 13th International Conference on Control Automation Robotics Vision (ICARCV), pages 1835-1840, Marina Bay Sands, Singapore, Dec 2014. 